\title{
Some New Gronwall-Bellman-Type Inequalities Based on the Modified Riemann-Liouville Fractional Derivative
}

\author{
Bin Zheng \\ School of Science, Shandong University of Technology, Zibo, Shandong 255049, China \\ Correspondence should be addressed to Bin Zheng; zhengbin2601@126.com
}

Received 22 May 2013; Accepted 28 August 2013

Academic Editor: Francisco J. Marcellán

Copyright (C) 2013 Bin Zheng. This is an open access article distributed under the Creative Commons Attribution License, which permits unrestricted use, distribution, and reproduction in any medium, provided the original work is properly cited.

By use of the properties of the modified Riemann-Liouville fractional derivative, some new Gronwall-Bellman-type inequalities are researched. First, we derive some new explicit bounds for the unknown functions lying in these inequalities, which are of different forms from some existing bounds in the literature. Then, we apply the results established to research the boundedness, uniqueness, and continuous dependence on the initial value for the solution to a certain fractional differential equation.

\section{Introduction}

During the past decades, a lot of integral and difference inequalities have been discovered, which play an important role in the research of the theory of differential, integral, and difference equations. In these inequalities, the GronwallBellman inequality and their generalizations have been paid much attention by many authors (e.g., see [1-22]), as such inequalities provide explicit bounds for the unknown functions concerned and can be used as a handy tool in the research of boundedness, global existence, uniqueness, stability, and continuous dependence of solutions to differential and integral equations as well as difference equations. However, most of the Gronwall-Bellman-type inequalities established so far can only be used in the research of differential equations of integer order, while in order to fulfill qualitative and quantitative analysis for solutions to some certain differential equations of fractional order, the earlier inequalities established are inadequate. So it is necessary to establish new inequalities so as to obtain the desired analysis for fractional differential equations.

In this paper, we establish some new Gronwall-Bellmantype inequalities. Based on some basic properties of the modified Riemann-Liouville fractional derivative, we derive explicit bounds for unknown functions concerned in these inequalities. The presented inequalities can be used as a handy tool in the qualitative as well as quantitative analysis of solutions to fractional differential equations.
The modified Riemann-Liouville fractional derivative, defined by Jumarie in [23-26], has many excellent characters in handling many fractional calculus problems. Many authors have investigated various applications of the modified Riemann-Liouville fractional derivative (e.g., see [27-33]). We now list the definition for it as follows.

Definition 1. The modified Riemann-Liouville derivative of order $\alpha$ is defined by the following expression:

$$
\begin{aligned}
& D_{t}^{\alpha} f(t) \\
& =\left\{\begin{array}{cl}
\frac{1}{\Gamma(1-\alpha)} \frac{d}{d t} \\
\times \int_{0}^{t}(t-\xi)^{-\alpha} \\
\times(f(\xi)-f(0)) d \xi, & 0<\alpha<1, \\
\left(f^{(n)}(t)\right)^{(\alpha-n)}, & n \leq \alpha<n+1, n \geq 1 .
\end{array}\right.
\end{aligned}
$$

Definition 2. The Riemann-Liouville fractional integral of order $\alpha$ on the interval $[0, t]$ is defined by

$$
\begin{aligned}
I^{\alpha} f(t) & =\frac{1}{\Gamma(1+\alpha)} \int_{0}^{t} f(s)(d s)^{\alpha} \\
& =\frac{1}{\Gamma(\alpha)} \int_{0}^{t}(t-s)^{\alpha-1} f(s) d s .
\end{aligned}
$$


Some important properties for the modified RiemannLiouville derivative and fractional integral are listed as follows (the interval concerned below is always defined by $[0, t]$ ):

(a) $D_{t}^{\alpha} t^{r}=(\Gamma(1+r) / \Gamma(1+r-\alpha)) t^{r-\alpha}$,

(b) $D_{t}^{\alpha}(f(t) g(t))=g(t) D_{t}^{\alpha} f(t)+f(t) D_{t}^{\alpha} g(t)$,

(c) $D_{t}^{\alpha} f[g(t)]=f_{g}^{\prime}[g(t)] D_{t}^{\alpha} g(t)=D_{g}^{\alpha} f[g(t)]\left(g^{\prime}(t)\right)^{\alpha}$,

(d) $I^{\alpha}\left(D_{t}^{\alpha} f(t)\right)=f(t)-f(0)$,

(e) $I^{\alpha}\left(g(t) D_{t}^{\alpha} f(t)\right)=f(t) g(t)-f(0) g(0)-I^{\alpha}(f(t)$ $\left.D_{t}^{\alpha} g(t)\right)$.

The next part of this paper is organized as follows. In Section 2, we present some new Gronwall-Bellman-type inequalities, and based on them we derive explicit bounds for unknown functions lying in these inequalities. In Section 3, for illustrating the validity of the established results, we apply them to research boundedness, uniqueness, and continuous dependence on initial data for the solution to a certain fractional differential equation, where the fractional derivative is defined in the sense of the modified Riemann-Liouville derivative. Finally, some summary comments are presented in Section 4.

\section{Main Results}

Theorem 3. Suppose that $u(t), a(t)$, and $b(t)$ are nonnegative continuous functions defined on $t \geq 0$. If the following inequality satisfies:

$$
u(t) \leq a(t)+\frac{b(t)}{\Gamma(\alpha)} \int_{0}^{t}(t-s)^{\alpha-1} u(s) d s, \quad t \geq 0,
$$

then, one has the following explicit estimate for $u(t)$ :

$$
\begin{aligned}
u(t) \leq & a(t)+b(t) \frac{1}{\Gamma(\alpha)} \\
& \times \exp \left[\int_{0}^{t^{\alpha} / \Gamma(1+\alpha)} b\left((s \Gamma(1+\alpha))^{1 / \alpha}\right) d s\right] \\
& \times \int_{0}^{t}(t-\tau)^{\alpha-1} a(\tau) \\
& \quad \times \exp \left[-\int_{0}^{\tau^{\alpha} / \Gamma(1+\alpha)} b\left((s \Gamma(1+\alpha))^{1 / \alpha}\right) d s\right] d \tau,
\end{aligned}
$$

Proof. Let $v(t)=(1 / \Gamma(\alpha)) \int_{0}^{t}(t-s)^{\alpha-1} u(s) d s$. Then, we have

$$
u(t) \leq a(t)+b(t) v(t), \quad t \geq 0 .
$$

Furthermore, as $u(t)$ is continuous, there exists $M$ such that $|u(t)| \leq M$ for $t \in[0, T]$, where $T>0$. So, for $t \in[0, T]$, we have $|v(t)| \leq(M / \Gamma(\alpha)) \int_{0}^{t}(t-s)^{\alpha-1} d s=(M / \Gamma(\alpha))\left(t^{\alpha} / \alpha\right)$, which implies that $v(0)=0$. So,

$$
D_{t}^{\alpha} v(t)=u(t) \leq a(t)+b(t) v(t), \quad t \geq 0 .
$$

Then, by the properties $(b)$ and $(c)$, we get that

$$
\begin{aligned}
D_{t}^{\alpha}\left\{v(t) \exp \left[-\int_{0}^{t^{\alpha} / \Gamma(1+\alpha)} b\left((s \Gamma(1+\alpha))^{1 / \alpha}\right) d s\right]\right\} \\
=\exp \left[-\int_{0}^{t^{\alpha} / \Gamma(1+\alpha)} b\left((s \Gamma(1+\alpha))^{1 / \alpha}\right) d s\right] D_{t}^{\alpha} v(t) \\
\quad+v(t) D_{t}^{\alpha}\left\{\exp \left[-\int_{0}^{t^{\alpha} / \Gamma(1+\alpha)} b\left((s \Gamma(1+\alpha))^{1 / \alpha}\right) d s\right]\right\} \\
=\exp \left[-\int_{0}^{t^{\alpha} / \Gamma(1+\alpha)} b\left((s \Gamma(1+\alpha))^{1 / \alpha}\right) d s\right] D_{t}^{\alpha} v(t) \\
\quad-b(t) v(t) \exp \left[-\int_{0}^{t^{\alpha} / \Gamma(1+\alpha)} b\left((s \Gamma(1+\alpha))^{1 / \alpha}\right) d s\right] \\
\quad \times D_{t}^{\alpha}\left(\frac{t^{\alpha}}{\Gamma(1+\alpha)}\right) .
\end{aligned}
$$

Since by (a) it holds that $D_{t}^{\alpha}\left(t^{\alpha} / \Gamma(1+\alpha)\right)=1$, then furthermore, we have

$$
\begin{aligned}
D_{t}^{\alpha} & \left\{v(t) \exp \left[-\int_{0}^{t^{\alpha} / \Gamma(1+\alpha)} b\left((s \Gamma(1+\alpha))^{1 / \alpha}\right) d s\right]\right\} \\
= & \exp \left[-\int_{0}^{t^{\alpha} / \Gamma(1+\alpha)} b\left((s \Gamma(1+\alpha))^{1 / \alpha}\right) d s\right] \\
& \times\left[D_{t}^{\alpha} v(t)-b(t) v(t)\right] \\
\leq & a(t) \exp \left[-\int_{0}^{t^{\alpha} / \Gamma(1+\alpha)} b\left((s \Gamma(1+\alpha))^{1 / \alpha}\right) d s\right] .
\end{aligned}
$$

Substituting $t$ with $\tau$ and fulfilling fractional integral of order $\alpha$ for (8) with respect to $\tau$ from 0 to $t$ we deduce that

$$
\begin{aligned}
& v(t) \exp \left\{-\int_{0}^{t^{\alpha} / \Gamma(1+\alpha)} b\left((s \Gamma(1+\alpha))^{1 / \alpha}\right) d s\right\} \\
& \leq \frac{1}{\Gamma(\alpha)} \int_{0}^{t}(t-\tau)^{\alpha-1} a(\tau) \\
& \quad \times \exp \left[-\int_{0}^{\tau^{\alpha} / \Gamma(1+\alpha)} b\left((s \Gamma(1+\alpha))^{1 / \alpha}\right) d s\right] d \tau,
\end{aligned}
$$

which implies that

$$
\begin{aligned}
v(t) \leq & \frac{1}{\Gamma(\alpha)} \exp \left[\int_{0}^{t^{\alpha} / \Gamma(1+\alpha)} b\left((s \Gamma(1+\alpha))^{1 / \alpha}\right) d s\right] \\
& \times \int_{0}^{t}(t-\tau)^{\alpha-1} a(\tau) \\
& \quad \times \exp \left\{-\int_{0}^{\tau^{\alpha} / \Gamma(1+\alpha)} b\left((s \Gamma(1+\alpha))^{1 / \alpha}\right) d s\right\} d \tau .
\end{aligned}
$$

Combining (5) and (10), we get (4). 
Remark 4. In Theorem 3, we note that the bound for $u(t)$ established in (4) is essentially different from that in [34, Theorem 1]. The bound in [34, Theorem 1] is expressed in a series, whose convergence has not been proved in fact, while the bound in (4) here is more explicit and easy to use. Moreover, the function $b(t)$ here is not necessarily nondecreasing, which is a generalization of the condition in [34, Theorem 1]. Besides, the result established here has been derived based on the modified Riemann-Liouville derivative, which is also different from [34].

Theorem 5. Suppose that $u(t), a(t)$, and $b(t)$ are defined as in Theorem 3. If the following inequality satisfies:

$$
u(t) \leq a(t)+\frac{b(t)}{\Gamma(\alpha)} \int_{0}^{t}(t-s)^{\alpha-1} s^{1-\alpha} u(s) d s, \quad t \geq 0
$$

then, one has the following explicit estimate for $u(t)$ :

$$
\begin{aligned}
& u(t) \leq a(t) \\
& +b(t) \frac{1}{\Gamma(\alpha)} \exp \left[(\Gamma(1+\alpha))^{(1-\alpha) / \alpha}\right. \\
& \times \int_{0}^{t^{\alpha} / \Gamma(1+\alpha)} s^{(1-\alpha) / \alpha} \\
& \left.\times b\left((s \Gamma(1+\alpha))^{1 / \alpha}\right) d s\right] \\
& \times \int_{0}^{t}(t-\tau)^{\alpha-1} \tau^{1-\alpha} a(\tau) \\
& \times \exp \left[-(\Gamma(1+\alpha))^{(1-\alpha) / \alpha}\right. \\
& \times \int_{0}^{\tau^{\alpha} / \Gamma(1+\alpha)} s^{(1-\alpha) / \alpha} \\
& \left.\times b\left((s \Gamma(1+\alpha))^{1 / \alpha}\right) d s\right] d \tau, \\
& t \geq 0 \text {. }
\end{aligned}
$$

Proof. Let $v(t)=(1 / \Gamma(\alpha)) \int_{0}^{t}(t-s)^{\alpha-1} s^{1-\alpha} u(s) d s$. Then, we have

$$
u(t) \leq a(t)+b(t) v(t), \quad t \geq 0 .
$$

Considering that $v(t)=(1 / \Gamma(\alpha)) \int_{0}^{t}(t-s)^{\alpha-1} s^{1-\alpha} u(s) d s=$ $(1 / \Gamma(\alpha)) t \int_{0}^{1}(1-z)^{\alpha-1} z^{1-\alpha} u(t z) d z$ and $u(t)$ is continuous satisfying $|u(t)| \leq M, t \in[0, T]$, where $M$ is a positive constant, we obtain

$$
\begin{array}{r}
|v(t)| \leq \frac{M}{\Gamma(\alpha)} t\left|\int_{0}^{1}(1-z)^{\alpha-1} z^{1-\alpha} d z\right|=M \Gamma(2-\alpha) t \\
t \in[0, T] .
\end{array}
$$

So, we have $v(0)=0$, and furthermore,

$$
D_{t}^{\alpha} v(t)=t^{1-\alpha} u(t) \leq t^{1-\alpha} a(t)+b(t) t^{1-\alpha} v(t), \quad t \geq 0 .
$$

Multiplying $\exp \left[-(\Gamma(1+\alpha))^{(1-\alpha) / \alpha} \int_{0}^{t^{\alpha} / \Gamma(1+\alpha)} s^{(1-\alpha) / \alpha} b((s \Gamma(1+\right.$ $\left.\alpha))^{1 / \alpha}\right) d s$ ] on both sides of (15), we get that

$$
\begin{aligned}
& D_{t}^{\alpha}\left\{v ( t ) \operatorname { e x p } \left[-(\Gamma(1+\alpha))^{(1-\alpha) / \alpha}\right.\right. \\
& \left.\left.\times \int_{0}^{t^{\alpha} / \Gamma(1+\alpha)} s^{(1-\alpha) / \alpha} b\left((s \Gamma(1+\alpha))^{1 / \alpha}\right) d s\right]\right\} \\
& =\exp \left[-(\Gamma(1+\alpha))^{(1-\alpha) / \alpha}\right. \\
& \left.\times \int_{0}^{t^{\alpha} / \Gamma(1+\alpha)} s^{(1-\alpha) / \alpha} b\left((s \Gamma(1+\alpha))^{1 / \alpha}\right) d s\right] D_{t}^{\alpha} v(t) \\
& +v(t) D_{t}^{\alpha}\left\{\operatorname { e x p } \left[-(\Gamma(1+\alpha))^{(1-\alpha) / \alpha}\right.\right. \\
& \times \int_{0}^{t^{\alpha} / \Gamma(1+\alpha)} s^{(1-\alpha) / \alpha} \\
& \left.\left.\times b\left((s \Gamma(1+\alpha))^{1 / \alpha}\right) d s\right]\right\} \\
& =\exp \left[-(\Gamma(1+\alpha))^{(1-\alpha) / \alpha}\right. \\
& \times \int_{0}^{t^{\alpha} / \Gamma(1+\alpha)} s^{(1-\alpha) / \alpha} \\
& \left.\times b\left((s \Gamma(1+\alpha))^{1 / \alpha}\right) d s\right] D_{t}^{\alpha} v(t) \\
& -t^{1-\alpha} b(t) v(t) \exp \left[-(\Gamma(1+\alpha))^{(1-\alpha) / \alpha}\right. \\
& \times \int_{0}^{t^{\alpha} / \Gamma(1+\alpha)} s^{(1-\alpha) / \alpha} \\
& \left.\times b\left((s \Gamma(1+\alpha))^{1 / \alpha}\right) d s\right] \\
& \times D_{t}^{\alpha}\left(\frac{t^{\alpha}}{\Gamma(1+\alpha)}\right)
\end{aligned}
$$


Since $D_{t}^{\alpha}\left(t^{\alpha} / \Gamma(1+\alpha)\right)=1$, then furthermore, we have

$$
\begin{aligned}
& D_{t}^{\alpha}\left\{v ( t ) \operatorname { e x p } \left[-(\Gamma(1+\alpha))^{(1-\alpha) / \alpha}\right.\right. \\
& \times \int_{0}^{t^{\alpha} / \Gamma(1+\alpha)} s^{(1-\alpha) / \alpha} \\
& \left.\left.\times b\left((s \Gamma(1+\alpha))^{1 / \alpha}\right) d s\right]\right\} \\
& =\exp \left[-(\Gamma(1+\alpha))^{(1-\alpha) / \alpha}\right. \\
& \times \int_{0}^{t^{\alpha} / \Gamma(1+\alpha)} s^{(1-\alpha) / \alpha} \\
& \left.\times b\left((s \Gamma(1+\alpha))^{1 / \alpha}\right) d s\right] \\
& \times\left[D_{t}^{\alpha} v(t)-t^{1-\alpha} b(t) v(t)\right] \\
& \leq t^{1-\alpha} a(t) \exp \left[-(\Gamma(1+\alpha))^{(1-\alpha) / \alpha}\right. \\
& \times \int_{0}^{t^{\alpha} / \Gamma(1+\alpha)} s^{(1-\alpha) / \alpha} \\
& \left.\times b\left((s \Gamma(1+\alpha))^{1 / \alpha}\right) d s\right] .
\end{aligned}
$$

Using $v(0)=0$, substituting $t$ with $\tau$, and fulfilling fractional integral of order $\alpha$ for (17) with respect to $\tau$ from 0 to $t$, we deduce that

$$
\begin{gathered}
v(t) \exp \left[-(\Gamma(1+\alpha))^{(1-\alpha) / \alpha} \int_{0}^{t^{\alpha} / \Gamma(1+\alpha)} s^{(1-\alpha) / \alpha}\right. \\
\left.\times b\left((s \Gamma(1+\alpha))^{1 / \alpha}\right) d s\right] \\
\leq \frac{1}{\Gamma(\alpha)} \int_{0}^{t}(t-\tau)^{\alpha-1} \tau^{1-\alpha} a(\tau) \\
\times \exp \left[-(\Gamma(1+\alpha))^{(1-\alpha) / \alpha}\right. \\
\times \int_{0}^{\tau^{\alpha} / \Gamma(1+\alpha)} s^{(1-\alpha) / \alpha} \\
\left.\times b\left((s \Gamma(1+\alpha))^{1 / \alpha}\right) d s\right] d \tau,
\end{gathered}
$$

which implies that

$$
\begin{array}{r}
v(t) \leq \frac{1}{\Gamma(\alpha)} \exp \left[(\Gamma(1+\alpha))^{(1-\alpha) / \alpha}\right. \\
\left.\times \int_{0}^{t^{\alpha} / \Gamma(1+\alpha)} s^{(1-\alpha) / \alpha} b\left((s \Gamma(1+\alpha))^{1 / \alpha}\right) d s\right] \\
\times \int_{0}^{t}(t-\tau)^{\alpha-1} \tau^{1-\alpha} a(\tau) \\
\times \exp \left[-(\Gamma(1+\alpha))^{(1-\alpha) / \alpha}\right. \\
\quad \times \int_{0}^{\tau^{\alpha} / \Gamma(1+\alpha)} s^{(1-\alpha) / \alpha} \\
\left.\times b\left((s \Gamma(1+\alpha))^{1 / \alpha}\right) d s\right] d \tau .
\end{array}
$$

Combining (13) and (19), we get (12).

Theorem 6. Suppose that $u(t), a(t)$, and $b(t)$ are defined as in Theorem 3, and furthermore, assume that $a(t)$ and $b(t)$ are both nondecreasing. $m(t)$ is nonnegative continuous on $t \geq 0$. If the following inequality satisfies:

$$
\begin{aligned}
u(t) \leq & a(t)+\int_{0}^{t} m(s) u(s) d s \\
& +\frac{b(t)}{\Gamma(\alpha)} \int_{0}^{t}(t-s)^{\alpha-1} u(s) d s, \quad t \geq 0
\end{aligned}
$$

then, for $t \geq 0$, one has the following explicit estimate for $u(t)$ :

$$
\begin{aligned}
u(t) \leq & \exp \left(\int_{0}^{t} m(s) d s\right) \\
\times & \left\{a(t)+b(t) \exp \left(\int_{0}^{t} m(\tau) d \tau\right) \frac{1}{\Gamma(\alpha)}\right. \\
& \times \exp \left[\int_{0}^{t^{\alpha} / \Gamma(1+\alpha)} \exp \left(\int_{0}^{(s \Gamma(1+\alpha))^{1 / \alpha}} m(\tau) d \tau\right)\right. \\
& \times \int_{0}^{t}(t-\tau)^{\alpha-1} a(\tau) \\
& \times \exp \left[-\int_{0}^{\tau^{\alpha} / \Gamma(1+\alpha)} \exp \left(\int_{0}^{(s \Gamma(1+\alpha))^{1 / \alpha}} m(\xi) d \xi\right)\right. \\
& \left.\left.\times b(1+\alpha)]^{1 / \alpha}\right) d s\right]
\end{aligned}
$$


Proof. Denote $z(t)=a(t)+(b(t) / \Gamma(\alpha)) \int_{0}^{t}(t-s)^{\alpha-1} u(s) d s$. Then,

$$
u(t) \leq z(t)+\int_{0}^{t} m(s) u(s) d s .
$$

Since $a(t)$ and $b(t)$ are both nondecreasing, then $z(t)$ is also nondecreasing, and so, we obtain that

$$
u(t) \leq z(t) \exp \left(\int_{0}^{t} m(s) d s\right) .
$$

So,

$$
\begin{aligned}
z(t) \leq & a(t)+\frac{b(t)}{\Gamma(\alpha)} \int_{0}^{t}(t-s)^{\alpha-1} z(s) \\
& \times \exp \left(\int_{0}^{s} m(\tau) d \tau\right) d s \\
\leq & a(t)+\frac{b(t)}{\Gamma(\alpha)} \exp \left(\int_{0}^{t} m(\tau) d \tau\right) \\
& \times \int_{0}^{t}(t-s)^{\alpha-1} z(s) d s .
\end{aligned}
$$

Notice that the structure of (24) is similar to that of inequality (3). So, a suitable application of Theorem 3 to (24) yields that

$$
\begin{aligned}
z(t) \leq & a(t) \\
& +b(t) \exp \left(\int_{0}^{t} m(\tau) d \tau\right) \frac{1}{\Gamma(\alpha)} \\
& \times \exp \left[\int_{0}^{t^{\alpha} / \Gamma(1+\alpha)} \exp \left(\int_{0}^{(s \Gamma(1+\alpha))^{1 / \alpha}} m(\tau) d \tau\right)\right. \\
& \times \int_{0}^{t}(t-\tau)^{\alpha-1} a(\tau) \\
& \times \exp \left[-\int_{0}^{\tau^{\alpha} / \Gamma(1+\alpha)} \exp \left(\int_{0}^{(s \Gamma(1+\alpha))^{1 / \alpha}} m(\xi) d \xi\right)\right.
\end{aligned}
$$

Combining (23) and (25), we can obtain the desired result.

Theorem 7. Suppose that $u(t), a(t)$, and $b(t)$ are defined as in Theorem 3, and furthermore, assume that $a(t)$ and $b(t)$ are both nondecreasing. $m(t)$ is nonnegative continuous on $t \geq 0$. If the following inequality satisfies:

$$
\begin{aligned}
u(t) \leq & a(t)+\int_{0}^{t} m(s) u(s) d s \\
& +\frac{b(t)}{\Gamma(\alpha)} \int_{0}^{t}(t-s)^{\alpha-1} s^{1-\alpha} u(s) d s, \quad t \geq 0,
\end{aligned}
$$

then, for $\alpha \geq 1$ and $t \geq 0$, one has the following explicit estimate for $u(t)$ :

$$
\begin{aligned}
& u(t) \leq \exp \left(\int_{0}^{t} m(s) d s\right) \\
& \times\left\{a(t)+b(t) \exp \left(\int_{0}^{t} m(\tau) d \tau\right) \frac{1}{\Gamma(\alpha)}\right. \\
& \times \exp \left[(\Gamma(1+\alpha))^{(1-\alpha) / \alpha}\right. \\
& \times \int_{0}^{t^{\alpha} / \Gamma(1+\alpha)} s^{(1-\alpha) / \alpha} \\
& \times \exp \left(\int_{0}^{(s \Gamma(1+\alpha))^{1 / \alpha}} m(\tau) d \tau\right) \\
& \left.\times b\left((s \Gamma(1+\alpha))^{1 / \alpha}\right) d s\right] \\
& \times \int_{0}^{t}(t-\tau)^{\alpha-1} \tau^{1-\alpha} a(\tau) \\
& \times \exp \left[-(\Gamma(1+\alpha))^{(1-\alpha) / \alpha}\right. \\
& \times \int_{0}^{\tau^{\alpha} / \Gamma(1+\alpha)} s^{(1-\alpha) / \alpha} \\
& \times \exp \left(\int_{0}^{(s \Gamma(1+\alpha))^{1 / \alpha}} m(\xi) d \xi\right) \\
& \left.\left.\times b\left((s \Gamma(1+\alpha))^{1 / \alpha}\right) d s\right] d \tau\right\} .
\end{aligned}
$$

The proof of Theorem 7 is similar to that of Theorem 6, except that Theorem 5 instead of Theorem 3 is applied at the end of the proof.

\section{Applications}

In this section, we will show that the inequalities established above are useful in the research of boundedness, uniqueness, and continuous dependence on the initial value for solutions to fractional differential equations. Consider the following fractional differential equation:

$$
D_{t}^{0.5} u(t)=f(t, u(t)), \quad t \geq 0,
$$

with the initial condition $u(0)=C$, where $f \in C(R \times R, R)$.

Theorem 8. Suppose that $u(t)$ is a solution of (28). If $|f(t, u)| \leq \sqrt{t}|u|$, then one has the following estimate for $u(t)$ :

$$
|u(t)| \leq|C|\left[1+\Gamma(1.5) \exp \left(\frac{t}{2 \Gamma(1.5)}\right) t\right], \quad t \geq 0 .
$$


Proof. Similar to [24, Equation (5.1)], we can obtain the equivalent integral form of (28) with the initial condition $u(0)=C$ as follows:

$$
u(t)=C+\frac{1}{\Gamma(0.5)} \int_{0}^{t}(t-s)^{-0.5} f(s, u(s)) d s .
$$

So,

$$
|u(t)| \leq|C|+\frac{1}{\Gamma(0.5)} \int_{0}^{s}(t-s)^{-0.5} \sqrt{s}|u(s)| d s .
$$

Then, a suitable application of Theorem 5 to (31) (with $\alpha=$ $0.5)$ yields

$$
\begin{aligned}
|u(t)| \leq|C|+\frac{|C|}{\Gamma(0.5)} \exp \left(\frac{t}{2 \Gamma(1.5)}\right) & \\
& \times \int_{0}^{t}(t-\tau)^{-0.5} \sqrt{\tau} \exp \left(-\frac{\tau}{2 \Gamma(1.5)}\right) d \tau, \\
t & \geq 0 .
\end{aligned}
$$

Since $\exp (-\tau / 2 \Gamma(1.5)) d \tau \leq 1$, then furthermore, we have

$$
\begin{aligned}
|u(t)| & \leq|C|+\frac{|C|}{\Gamma(0.5)} \exp \left(\frac{t}{2 \Gamma(1.5)}\right) \int_{0}^{t}(t-\tau)^{-0.5} \sqrt{\tau} d \tau \\
& =|C|+\frac{|C|}{\Gamma(0.5)} \exp \left(\frac{t}{2 \Gamma(1.5)}\right) B(1.5,0.5) t \\
& \leq|C|\left[1+\Gamma(1.5) \exp \left(\frac{t}{2 \Gamma(1.5)}\right) t\right], \quad t \geq 0,
\end{aligned}
$$

which is the desired result.

Remark 9. In Theorem 8 , if $f(t, u)$ satisfies $|f(t, u)| \leq K u$ instead, then one can apply Theorem 3 instead of Theorem 5 in the proof and obtain the following estimate for $u(t)$ :

$$
\begin{aligned}
u(t) \leq|C| & \left\{1+\frac{1}{\Gamma(0.5)} \exp \left(\frac{\sqrt{t}}{\Gamma(1.5)}\right)\right. \\
& \left.\times \int_{0}^{t}(t-\tau)^{-0.5} \exp \left[-\frac{\sqrt{\tau}}{\Gamma(1.5)}\right] d \tau\right\}, \quad t \geq 0 .
\end{aligned}
$$

Furthermore, we have

$$
u(t) \leq|C|\left[1+\frac{2}{\Gamma(0.5)} \sqrt{t} \exp \left(\frac{\sqrt{t}}{\Gamma(1.5)}\right)\right], \quad t \geq 0 .
$$

Theorem 10. If $|f(t, u)-f(t, v)| \leq \sqrt{t}|u-v|$, then (28) has a unique solution.

Proof. Suppose that (28) has two solutions $u_{1}(t)$ and $u_{2}(t)$. Then, similar to Theorem 8 , we can obtain

$$
\begin{aligned}
& u_{1}(t)=C+\frac{1}{\Gamma(0.5)} \int_{0}^{t}(t-s)^{-0.5} f\left(s, u_{1}(s)\right) d s, \\
& u_{2}(t)=C+\frac{1}{\Gamma(0.5)} \int_{0}^{t}(t-s)^{-0.5} f\left(s, u_{2}(s)\right) d s .
\end{aligned}
$$

Furthermore,

$$
\begin{aligned}
u_{1}(t)-u_{2}(t)=\frac{1}{\Gamma(0.5)} \int_{0}^{t}(t-s)^{-0.5} & \\
& \times\left[f\left(s, u_{1}(s)\right)-f\left(s, u_{2}(s)\right)\right] d s
\end{aligned}
$$

which implies that

$$
\begin{aligned}
\left|u_{1}(t)-u_{2}(t)\right| \leq \frac{1}{\Gamma(0.5)} \int_{0}^{t}(t-s)^{-0.5} & \quad \times\left|f\left(s, u_{1}(s)\right)-f\left(s, u_{2}(s)\right)\right| d s \\
\leq & \frac{1}{\Gamma(0.5)} \int_{0}^{t}(t-s)^{-0.5} \sqrt{s}\left|u_{1}(s)-u_{2}(s)\right| d s .
\end{aligned}
$$

Treating $\left|u_{1}(t)-u_{2}(t)\right|$ as one independent function and applying Theorem 5 to (38) (with $\alpha=0.5$ ), we get that $\left|u_{1}(t)-u_{2}(t)\right| \leq 0$, which implies that $u_{1}(t) \equiv u_{2}(t)$. So, the proof is complete.

Now we research the continuous dependence on the initial value for the solution of (28).

Theorem 11. Under the conditions of Theorem 10, the solution of (28) depends continuously on the initial value of $t \in[0, T]$, where $T$ is arbitrarily large.

Proof. Let $u(t)$ be the solution of (28), and let $\widetilde{u}(t)$ be the solution of the following fractional differential equation:

$$
D_{t}^{0.5} \widetilde{u}(t)=f(t, \widetilde{u}(t)), \quad t \geq 0
$$

with the initial condition $\widetilde{u}(0)=\widetilde{C}$.

Similar to Theorem 8, we get that

$$
\widetilde{u}(t)=C+\frac{1}{\Gamma(0.5)} \int_{0}^{t}(t-s)^{-0.5} f(s, \widetilde{u}(s)) d s .
$$

So, we have

$$
\begin{aligned}
u(t)-\widetilde{u}(t)= & C-\widetilde{C} \\
& +\frac{1}{\Gamma(0.5)} \int_{0}^{t}(t-s)^{-0.5} \\
& \quad \times[f(s, u(s))-f(s, \tilde{u}(s))] d s,
\end{aligned}
$$


which implies that

$$
\begin{aligned}
|u(t)-\tilde{u}(t)| \leq & |C-\widetilde{C}| \\
& +\frac{1}{\Gamma(0.5)} \int_{0}^{t}(t-s)^{-0.5} \\
\leq & |C-\widetilde{C}| f(s, u(s))-f(s, \widetilde{u}(s)) \mid d s \\
& +\frac{1}{\Gamma(0.5)} \int_{0}^{t}(t-s)^{-0.5} \sqrt{s}|u(s)-\widetilde{u}(s)| d s .
\end{aligned}
$$

Applying Theorem 5 to (42), after some basic computation, we can get that

$$
|u(t)-\widetilde{u}(t)| \leq|C-\widetilde{C}|\left[1+\frac{\Gamma(1.5)}{\Gamma(2)} \exp \left(\frac{t}{2 \Gamma(1.5)}\right) t\right] .
$$

From (43), one can see that small change in the initial value leads to small change in the solution on the closed interval $[0, T]$. So, the proof is complete.

\section{Conclusions}

We have presented some new Gronwall-Bellman-type inequalities, and based on them we derived explicit bounds for the unknown functions concerned, which are different from the existing bounds in the literature. As one can see, the results established are useful in fulfilling qualitative and quantitative analyses such as the boundedness, uniqueness, and continuous dependence on the initial value and parameter for solutions to certain fractional differential equations. Finally, we note that the method used in Theorems 3 and 5 is of generality and can be used to establish other Gronwall-Bellman-type inequalities with more complicated and general forms, which are expected to further research.

\section{Conflict of Interests}

The author declares that there is no conflict of interests regarding the publication of this paper.

\section{Acknowledgment}

The author would like to thank the reviewers very much for their valuable suggestions on improving this paper.

\section{References}

[1] T. H. Gronwall, "Note on the derivatives with respect to a parameter of the solutions of a system of differential equations," Annals of Mathematics, vol. 20, no. 4, pp. 292-296, 1919.

[2] R. Bellman, "The stability of solutions of linear differential equations,” Duke Mathematical Journal, vol. 10, pp. 643-647, 1943.
[3] Q. Feng and B. Zheng, "Generalized Gronwall-Bellman-type delay dynamic inequalities on time scales and their applications," Applied Mathematics and Computation, vol. 218, no. 15, pp. 7880-7892, 2012.

[4] Q. H. Ma, "Estimates on some power nonlinear VolterraFredholm type discrete inequalities and their applications," Journal of Computational and Applied Mathematics, vol. 233, no. 9, pp. 2170-2180, 2010.

[5] W. S. Wang, "A class of retarded nonlinear integral inequalities and its application in nonlinear differential-integral equation," Journal of Inequalities and Applications, vol. 2012, article 154, 10 pages, 2012.

[6] S. H. Saker, "Some nonlinear dynamic inequalities on time scales," Mathematical Inequalities \& Applications, vol. 14, no. 3, pp. 633-645, 2011.

[7] R. A. C. Ferreira and D. F. M. Torres, "Generalized retarded integral inequalities," Applied Mathematics Letters, vol. 22, no. 6, pp. 876-881, 2009.

[8] Q. Feng, F. Meng, and B. Zheng, "Gronwall-Bellman type nonlinear delay integral inequalities on time scales," Journal of Mathematical Analysis and Applications, vol. 382, no. 2, pp. 772784, 2011.

[9] O. Lipovan, "Integral inequalities for retarded Volterra equations," Journal of Mathematical Analysis and Applications, vol. 322, no. 1, pp. 349-358, 2006.

[10] S. H. Saker, "Some nonlinear dynamic inequalities on time scales and applications," Journal of Mathematical Inequalities, vol. 4, no. 4, pp. 561-579, 2010.

[11] B. Zheng, Q. Feng, F. Meng, and Y. Zhang, "Some new Gronwall-Bellman type nonlinear dynamic inequalities containing integration on infinite intervals on time scales," Journal of Inequalities and Applications, vol. 2012, article 201, 20 pages, 2012.

[12] B. G. Pachpatte, Inequalities for Differential and Integral Equations, Academic Press, New York, NY, USA, 1998.

[13] R. P. Agarwal, S. Deng, and W. Zhang, "Generalization of a retarded Gronwall-like inequality and its applications," Applied Mathematics and Computation, vol. 165, no. 3, pp. 599-612, 2005.

[14] W. S. Cheung and J. Ren, "Discrete non-linear inequalities and applications to boundary value problems," Journal of Mathematical Analysis and Applications, vol. 319, no. 2, pp. 708-724, 2006.

[15] W. N. Li, "Some delay integral inequalities on time scales," Computers \& Mathematics with Applications, vol. 59, no. 6, pp. 1929-1936, 2010.

[16] W. S. Wang, "Some retarded nonlinear integral inequalities and their applications in retarded differential equations," Journal of Inequalities and Applications, vol. 2012, article 75, 8 pages, 2012.

[17] Y. H. Kim, "Gronwall, Bellman and Pachpatte type integral inequalities with applications," Nonlinear Analysis A, vol. 71, no. 12, pp. e2641-e2656, 2009.

[18] W. N. Li, M. Han, and F. W. Meng, "Some new delay integral inequalities and their applications," Journal of Computational and Applied Mathematics, vol. 180, no. 1, pp. 191-200, 2005.

[19] Q. Feng, F. Meng, Y. Zhang, B. Zheng, and J. Zhou, "Some nonlinear delay integral inequalities on time scales arising in the theory of dynamics equations," Journal of Inequalities and Applications, vol. 2011, article 29, 14 pages, 2011.

[20] Q. Feng, F. Meng, and Y. Zhang, "Generalized GronwallBellman-type discrete inequalities and their applications," Journal of Inequalities and Applications, vol. 2011, article 47, 12 pages, 2011. 
[21] Z. Meng, B. Zheng, and C. Wen, "Some new integral inequalities on time scales containing integration on infinite intervals," Journal of Inequalities and Applications, vol. 2013, article 245, 2013.

[22] Y. Wang, Q. Feng, S. Zhou, and F. Xu, "Some new generalized Gronwall-Bellman-type integral inequalities in two independent variables on time scales," Journal of Inequalities and Applications, vol. 2013, article 234, 2013.

[23] G. Jumarie, "Modified Riemann-Liouville derivative and fractional Taylor series of nondifferentiable functions further results," Computers \& Mathematics with Applications, vol. 51, no. 9-10, pp. 1367-1376, 2006.

[24] G. Jumarie, "Table of some basic fractional calculus formulae derived from a modified Riemann-Liouville derivative for nondifferentiable functions," Applied Mathematics Letters, vol. 22, no. 3, pp. 378-385, 2009.

[25] G. Jumarie, "Cauchy's integral formula via the modified Riemann-Liouville derivative for analytic functions of fractional order," Applied Mathematics Letters, vol. 23, no. 12, pp. 1444-1450, 2010.

[26] G. Jumarie, "Fractional partial differential equations and modified Riemann-Liouville derivative new methods for solution," Journal of Applied Mathematics \& Computing, vol. 24, no. 1-2, pp. 31-48, 2007.

[27] S. Zhang and H. Q. Zhang, "Fractional sub-equation method and its applications to nonlinear fractional PDEs," Physics Letters A, vol. 375, no. 7, pp. 1069-1073, 2011.

[28] S. Guo, L. Mei, Y. Li, and Y. Sun, "The improved fractional sub-equation method and its applications to the space-time fractional differential equations in fluid mechanics," Physics Letters A, vol. 376, no. 4, pp. 407-411, 2012.

[29] B. Lu, "The first integral method for some time fractional differential equations," Journal of Mathematical Analysis and Applications, vol. 395, no. 2, pp. 684-693, 2012.

[30] B. Zheng, " $\left(G^{\prime} / G\right)$-expansion method for solving fractional partial differential equations in the theory of mathematical physics," Communications in Theoretical Physics, vol. 58, no. 5, pp. 623-630, 2012.

[31] Q. Feng, "Exact solutions for fractional differential-difference equations by an extended riccati sub-ODE method," Communications in Theoretical Physics, vol. 59, no. 5, pp. 521-527, 2013.

[32] G.- c. Wu and E. W. M. Lee, "Fractional variational iteration method and its application," Physics Letters A, vol. 374, no. 25, pp. 2506-2509, 2010.

[33] B. Tang, Y. He, L. Wei, and X. Zhang, "A generalized fractional sub-equation method for fractional differential equations with variable coefficients," Physics Letters A, vol. 376, no. 38-39, pp. 2588-2590, 2012.

[34] H. Ye, J. Gao, and Y. Ding, "A generalized Gronwall inequality and its application to a fractional differential equation," Journal of Mathematical Analysis and Applications, vol. 328, no. 2, pp. 1075-1081, 2007. 


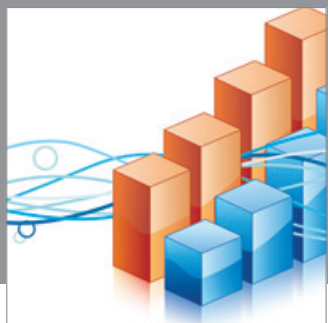

Advances in

Operations Research

mansans

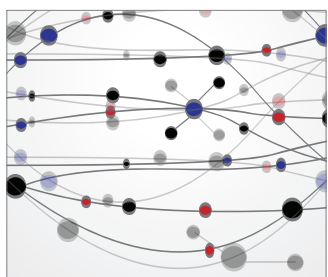

The Scientific World Journal
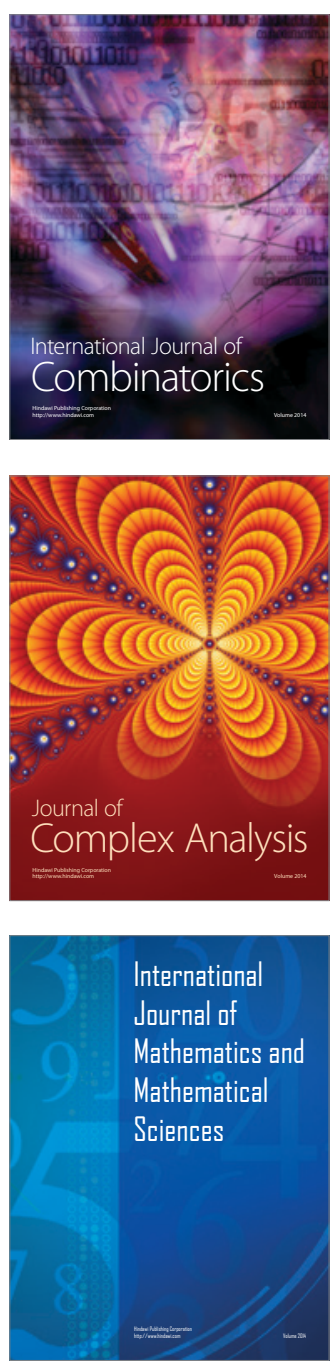
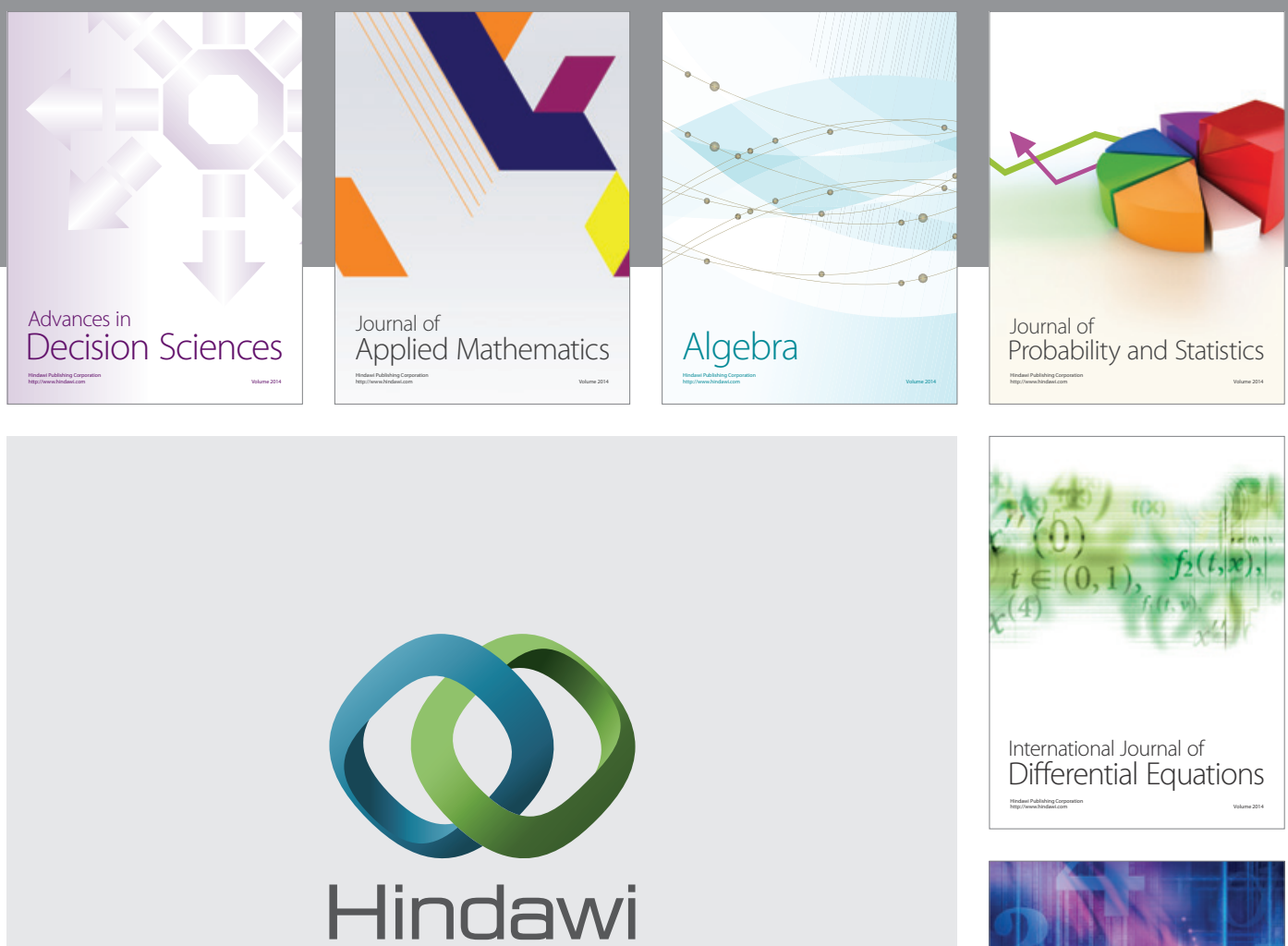

Submit your manuscripts at http://www.hindawi.com
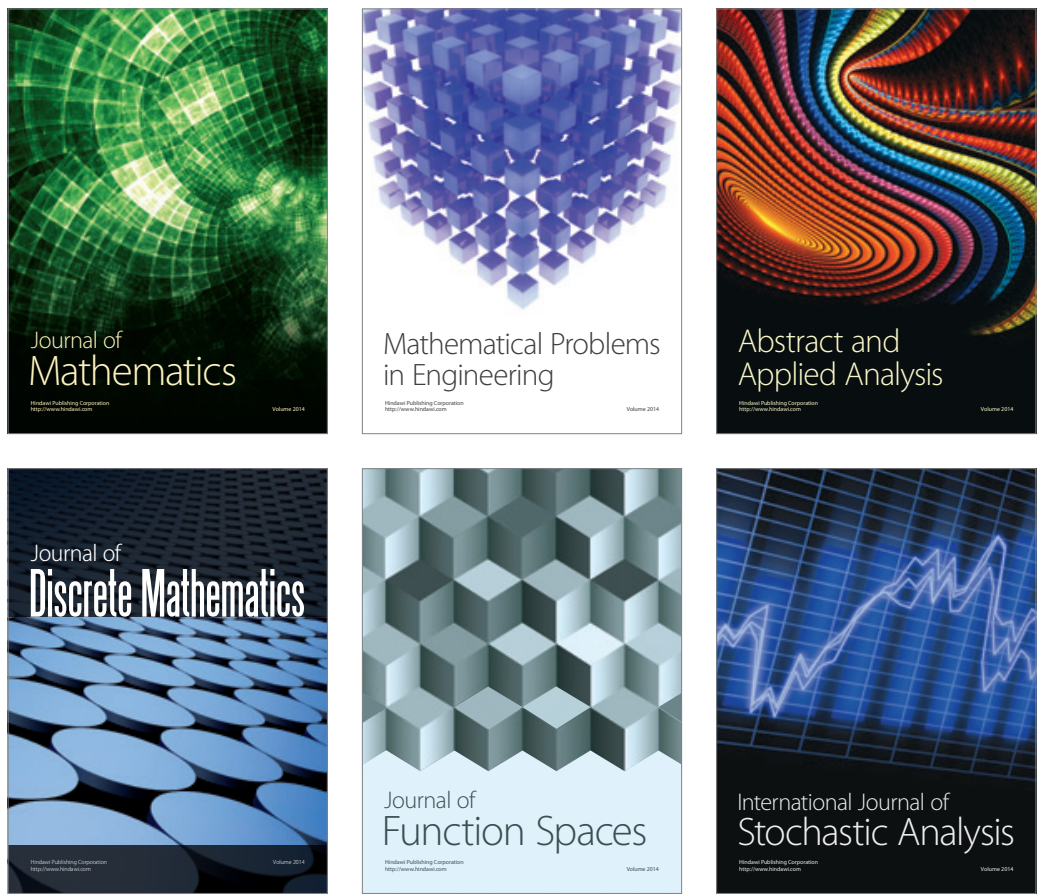

Journal of

Function Spaces

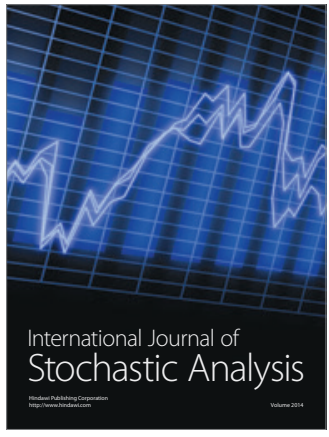

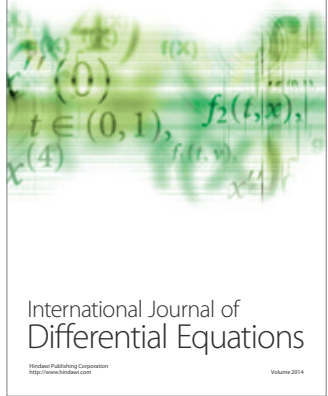
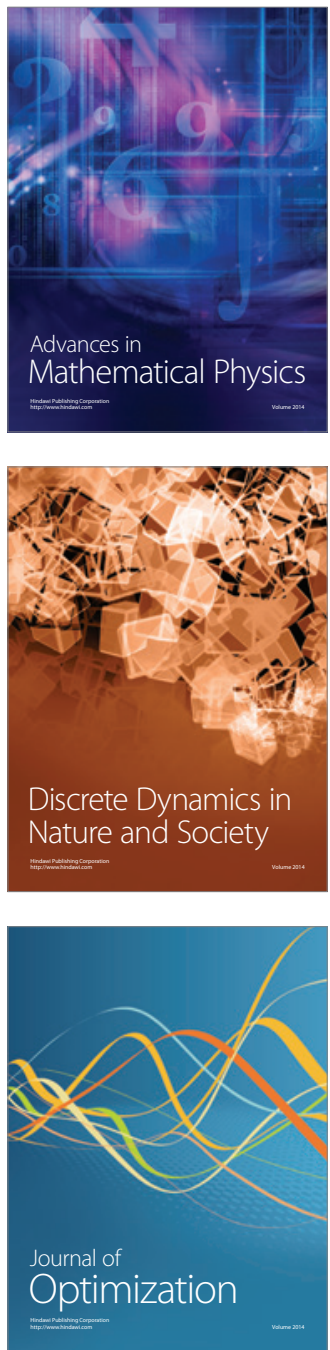